\title{
Assessment of two methods for rapid intrapartum detection of vaginal group B streptococcal colonisation
}

\author{
A J H Simpson, J A Mawn, S R Heard
}

\begin{abstract}
Aims-To compare two methods for the rapid detection of intrapartum vaginal carriage of group B streptococci (Streptococcus agalactiae) with standard culture techniques and to establish their suitability for routine use.
\end{abstract}

Methods-Vaginal swabs from 266 patients in labour were incubated in glucose broth in an anaerobic atmosphere for four to six hours. The Wellcogen Strep B latex particle agglutination test kit was subsequently used for antigen detection. In the second part of the study swabs from 117 women were assessed for the presence of group $B$ streptococci using the ICON STREP $B$ immunoconcentration assay (Hybritech). Both methods were compared with standard semiquantitative culture on Columbia horse blood agar and Islam's medium.

Results-In the first study vaginal carriage of group B streptococci was shown in 38 of $266(14 \cdot 3 \%)$ patients by culture. Latex particle agglutination with the Wellcogen kit detected 30 of these positive results (sensitivity $\mathbf{7 8 . 9 \%}$, specificity $100 \%$ ). In those patients with moderate to heavy colonisation ( $>10^{4}$ colony forming units per millilitre) antigen was detected in all (26/26) culture positive patients (sensitivity $100 \%$, specificity $100 \%$ ). In the second study $16(13 \cdot 7 \%)$ patients were culture positive. The ICON test detected 11 positive results (sensitivity $68 \cdot 8 \%$, specificity $100 \%$ ) and for heavy colonisation $\left(10^{5} \mathrm{cfu} / \mathrm{ml}\right)$ detected nine of nine cases (sensitivity $100 \%$, specificity $100 \%$ ). The ICON test took 10 to 15 minutes to perform.

Conclusion-These tests are potentially useful for the rapid detection of group B streptococci vaginal colonisation in labour, particularly heavy colonisation. Both tests are insufficiently sensitive to replace standard culture methods.

Department of

Medical Microbiology,

St Bartholomew's

Hospital Medical

College, West

Smithfield, London

EC1A 7BE

A J H Simpson

J A Mawn

S R Heard

Correspondence to:

Dr A J H Simpson

Accepted for publication

9 February 1994 (f Clin Pathol 1994;47:752-755)

Recent editorials in two leading journals have discussed the need for more rapid and reliable methods of intrapartum detection of vaginal carriage of group B streptococci. ${ }^{12}$ This organism remains an important cause of neonatal sepsis, causing significant mortality and morbidity, much of which is preventable. ${ }^{3}$ Ultimately an effective vaccine may become available, ${ }^{4}$ but until then antibiotics are the main means of limiting disease.

Chemoprophylaxis during labour is of undoubted benefit, ${ }^{5-9}$ but the problem is one of patient selection for such prophylaxis. Guidelines for chemoprophylaxis have recently been issued in the USA. ${ }^{10}$ Known carriers of group B streptococci with defined risk factors (maternal fever during labour, premature or prolonged rupture of membranes, multiple pregnancy or premature labour) are recommended to receive prophylaxis. Universal screening (by culture) during pregnancy will identify many carriers, but carriage is unstable. Some women found to be negative by screening during pregnancy will be positive at term, while others may receive insufficient antenatal care to be screened at all. Overreliance on antenatal screening will therefore place some women at continued risk. Selective screening has been shown to be unhelpful. ${ }^{11}$ The ideal time to screen is during labour, but conventional screening at this time is too slow if results are to guide intrapartum prophylaxis. Quicker results are needed.

Many published papers demonstrate the continuing search for a reliable test, ${ }^{12}$ but no test has yet become established. This paper reports the results of a comparison of two rapid methods with standard culture for intrapartum detection of vaginal group B streptococcal carriage.

\section{Methods}

The study was divided into two parts: part A involved enrichment of vaginal swabs, followed by latex particle agglutination (LPA), using the Wellcogen Strep B test (Wellcome Diagnostics, Dartford, Kent). Part B involved enrichment followed by use of the ICON STREP B test (Hybritech Europe SA, Liège, Belgium). Low vaginal swabs were obtained by the attending midwife, after informed consent, from women presenting to the delivery suite in labour at Homerton Hospital, Hackney, over a four month period. For the second part of the study duplicate swabs were taken from each patient. After sampling the cotton-tipped swabs were placed into Amies transport medium and sent to the laboratory.

\section{PART A}

Swabs were inoculated on to non-selective Oxoid Columbia horse blood agar and Islam's medium ${ }^{13}$ (five swabs per plate plus a positive control organism). Each swab was 
then inoculated into $3 \mathrm{ml} 0 \cdot 25 \%$ glucose broth (Southern Group). The tips of the swabs were cut and left in the broth. Plates were incubated at $37^{\circ} \mathrm{C}$ for up to 48 hours in an anaerobic atmosphere, and were examined for haemolysis or pigment production at 24 and 48 hours. Orange pigment production on Islam's medium was taken as evidence of presumptive group B streptococci. Haemolytic colonies were tested with the Streptex Lancefield grouping kit (Wellcome Diagnostics, Dartford, Kent) for group B streptococcal antigen. Quantitative growth of group B streptococci on the primary plates was assessed as follows.

\begin{tabular}{|ll|}
\hline Semi quantitative & Colony forming \\
assessment & units \\
0 & 0 \\
$1+$ & $<10$ \\
$2+$ & $10-50$ \\
$3+$ & $>50$ (or confluent growth) \\
\hline
\end{tabular}

The glucose broths were incubated at $37^{\circ} \mathrm{C}$ in an anaerobic atmosphere with the tops loosened so that maximum anaerobic conditions were obtained, for four to six hours. Swab tips were then removed and discarded. Each broth was then subcultured on to Islam's medium and incubated anaerobically. The broths were boiled in a water bath for five minutes and then centrifuged for five minutes at $3000 \mathrm{rpm}$. Latex testing of the supernatant fluid was then performed using the Wellcogen Strep B test (Wellcome Diagnostics). Thirty microlitres of broth was mixed with about $5 \mu \mathrm{l}$ (1 loopful) of latex suspension on a glass tile and the tile was rotated for up to two minutes. A control latex was used to eliminate samples causing autoagglutination. Positive results were taken as those which caused visible agglutination within two minutes.

Table 1 Comparison of plate counts from known group $B$ streptococcal concentrations

\begin{tabular}{lll}
\hline Colony count & Grade & $\begin{array}{l}\text { Equivalent group } B \\
\text { streptococcal concentration }(c f u / m l)\end{array}$ \\
\hline$<10$ & $1+$ & $10^{3}$ \\
$10-50$ & $2+$ & $10^{4}$ \\
$>50$ & $3+$ & $10^{5}$ \\
\hline
\end{tabular}

Table 2 Comparison of culture and LPA after pre-enrichment

\begin{tabular}{llcc}
\hline $\begin{array}{l}\text { Group B streptococci } \\
\text { culture result }\end{array}$ & No of patients & LPA positive & $\%$ LPA positive \\
\hline 0 & 228 & 0 & 0 \\
$1+$ & 12 & 4 & 33 \\
$2+$ & 7 & 7 & 100 \\
$3+$ & 19 & 19 & 100
\end{tabular}

${ }^{\star}$ LPA $=$ Latex particle agglutination test

For all culture positive results:

Sensitivity $=78.9 \%$

Specificity $=100 \%$

Positive predictive value $=100 \%$

Negative predictive value $=96.6 \%$

For moderate to heavily colonised patients $\left(2+\right.$ or $\left.3+,>10^{4} \mathrm{cfu} / \mathrm{ml}\right)$ :

Sensitivity $=100 \%$

Specificity $=100 \%$

Positive predictive value $=100 \%$

Negative predictive value $=100 \%$
PART B

Duplicate swabs were collected from each patient-one swab was processed as in part A. The second swab was processed using the ICON STREP B immunoconcentration assay as per the manufacturer's instructions. This test involves an enzyme immunoassay, with labelled anti-group B streptococcal antibody bound to a porous membrane within an ICON cylinder. Each swab was subjected to a nitrous acid antigen extraction procedure; the extract was mixed with alkaline phosphataseconjugated anti-group B streptococcal antibody. The filtered mixture was passed through the membrane containing bound group B streptococcal antibody, in the ICON cylinder. After washing and addition of enzyme substrate a colour change was seen in the centre of the ICON membrane if labelled group B streptococcal antibody-antigen complex had been bound to the membrane. A colour change of any intensity was taken as positive. Results were read after two minutes and designated either positive or negative-no grading of the result was possible.

An assessment of the sensitivity of these tests was performed by artificially inoculating swabs into broths containing serial dilutions of group B streptococci, prepared from an overnight culture, or by inoculating swabs with $100 \mu \mathrm{l}$ of each broth dilution. These were plated out, incubated, and colony counts graded as for clinical swabs. Broth viable counts were calculated by a spread plate method. A comparison of colony counts on swab plates with known group B streptococcal concentrations was made.

\section{Results}

A total of 266 patients were tested in part A of the study; 117 of these were also studied in part B. A total of 38 of $266(14.3 \%)$ patients were positive for group B streptococcal vaginal carriage by culture. For part B alone, 16 of $117(13.7 \%)$ patients were culture positive.

There were no cases of sepsis due to group B streptococci in infants born to mothers enrolled in the study.

In assessing the sensitivity of plate cultures, results were similar for both arms. Comparisons are shown in table 1 .

PART A

Thirty of 38 patients with group B streptococcal carriage detected by culture were detected by LPA testing after anaerobic preincubation in broth. There were no false positive results. Results are shown in table 2 .

PART B

Eleven of 16 culture positive patients were detected using the ICON test. There were no false positive results. Results are shown in table 3.

\section{Discussion}

Many rapid tests for the detection of group B streptococci have been studied, including 
Table 3 Comparison of culture and ICON immunoassay

\begin{tabular}{llll}
\hline $\begin{array}{l}\text { Group B streptococci } \\
\text { culture result }\end{array}$ & No of patients & ICON positive & $\%$ ICON positive \\
\hline 0 & 101 & 0 & 0 \\
$1+$ & 5 & 1 & 20 \\
$2+$ & 2 & 1 & 50 \\
$3+$ & 9 & 9 & 100 \\
\hline
\end{tabular}

For all culture positive patients:

Sensitivity $=68 \cdot 8 \%$

Specificity $=100 \%$

Positive predictive value $=100 \%$

Negative predictive value $=95.3 \%$

For heavily colonised patients $\left(3+,>10^{5} \mathrm{cfu} / \mathrm{ml}\right)$ :

Sensitivity $=100 \%$

Specificity $=100 \%$

Positive predictive value $=100 \%$

Negative predictive value $=100 \%$
Gram stains, LPA tests, coagglutination, pigment production, enzyme immunoassays (EIA) and DNA probes, without any one test becoming established as sufficiently reliable or sensitive. ${ }^{12}$ Most show improved sensitivity for heavily colonised patients.

Latex particle agglutination tests have been studied by many investigators, although most have looked at direct antigen extraction from vaginal swabs, followed by LPA. ${ }^{12115}$ A few have studied broth pre-enrichment followed by LPA. Stiller et al, using the Streptex kit, showed a sensitivity of $91.8 \%$ after eight to 12 hours of pre-incubation in Todd-Hewitt broth (specificity $97.6 \%$, positive predictive value $89.6 \%$, negative predictive value $98.2 \%) .{ }^{16}$ The Wellcogen kit has also been studied, demonstrating $86 \cdot 8 \%$ sensitivity after eight hours of pre-incubation, which increased to $94.7 \%$ after nitrous acid extraction of centrifuged pellets. ${ }^{17}$ Staphylococcal coagglutination tests have been reported to have low sensitivity after pre-enrichment. ${ }^{12} 18$ Our experience with the Wellcogen test shows that it is highly specific and has excellent sensitivity for moderate to heavy colonisation after four to six hours of anaerobic pre-enrichment, a shorter time than has been studied before. The sensitivity of the test, down to at least $10^{4}$ $\mathrm{cfu} / \mathrm{ml}$, is particularly noteworthy and makes it very attractive. However, this test, while being simple and very reliable, is only suitable for performance within a laboratory setting rather than at the bedside. The time taken to achieve a result is still a drawback, both in terms of rapidity and out of hours work, but a useful result could be provided during the period of labour in many cases. In a Finnish study, using an LPA test after pre-enrichment for five to seven hours, only $38 \%$ of patients had delivered before a test result could influence management. ${ }^{19}$

There have been two previous reports of experience with the ICON STREP B test. ${ }^{2021}$ Gentry et al, in a study of 300 patients in labour, compared the ICON with culture on blood agar. ${ }^{20}$ They showed a sensitivity of $33 \%$, a specificity of $95 \%$, a positive predictive value of $43 \%$ and a negative predictive value of $93 \%$. For heavily colonised patients, defined in this study as greater than $10^{5} \mathrm{cfu} / \mathrm{ml}$ of vaginal fluid, the sensitivity was $100 \%$. Armer et al showed a sensitivity for the ICON of only $11 \%$ overall in a study of 182 patients (specificity $100 \%$, positive predictive value $100 \%$, negative predictive value $78 \%$ ). ${ }^{21}$ The sensitivity was $100 \%$ for heavily colonised patients, again defined as $>10^{5} \mathrm{cfu} / \mathrm{ml}$. This latter study used broth enrichment, in addition to culture, for comparison, ${ }^{21}$ and thus detected many more culture positive cases $(23 \%)$ than either the previous study $(10 \%)^{20}$ or our investigation. This probably accounts for the much lower overall sensitivity. Both these previous studies estimated heaviness of colonisation by inoculating swabs with an arbitrary $100 \mu \mathrm{l}$ of broth containing group B streptococci at known titres and plating these out as for clinical swabs. This may have resulted in overestimates of the density of group B streptococci in vaginal fluid. However, the limit of sensitivity seemed to be similar in both reports, and our findings suggest that $100 \mu \mathrm{l}$ per swab gives similar results to direct inoculation into broth. Our results confirm the findings of these two studies, demonstrating that the ICON has a very high sensitivity and specificity for heavily colonised patients. It failed to detect some more lightly colonised patients, some of whose infants will become colonised, so it could not replace standard culture methods for detection of group B streptococci. However, the risk to an infant of group B streptococcal colonisation and sepsis is directly related to the heaviness of maternal vaginal carriage, ${ }^{22} 23$ so detection of heavily colonised mothers alone is of value. We also found that the ICON test had a high positive predictive value, unlike Gentry et al,,$^{20}$ but confirming the findings of Armer et al. ${ }^{21}$ The ICON test is easy to interpret and the above findings, together with the short time to produce a result, indicate that this test is potentially very useful as a tool for rapid testing, as an adjunct to plate culture. However, although rapid, there are several steps involved in performing the test, thereby possibly limiting its use to trained personnel and hence its usefulness as a bedside test.

Costs are an inevitable consideration. The Wellcogen Strep B test costs $£ 46$ ( + VAT) (at 1993 prices) and provides sufficient reagent for 200 tests by this method ( $£ 0 \cdot 23 /$ test), but the main expenses are staff costs in providing a test that takes up to six hours to perform, especially for out of hours provision. The ICON STREP B test costs $£ 65$ (+ VAT) for 20 tests ( $£ 3 \cdot 25 /$ test), but offers considerable savings in terms of laboratory staff time. Local considerations must influence which of these tests a laboratory might decide to offer.

Both of these tests are highly sensitive and specific for patients heavily colonised with group B streptococci. The ICON STREP B immunoconcentration assay is very quick and relatively simple. The Wellcogen Strep B test, although taking up to six hours with preenrichment, was more sensitive. Neither of these tests is sufficiently sensitive to replace standard culture methods, but both could be of value for rapid detection of maternal group B streptococcal carriage during labour. 
We thank all the staff of the delivery suite at Homerton Hospital for their assistance in this study. The ICON STREP B immunoassay was kindly provided by Hybritech Europe.

1 van Oppen C, Feldman R. Antibiotic prophylaxis of neonatal group B streptococcal infections. BMF 1993 306:411-2.

2 Wessels MR, Kasper DL. The changing face of Group B streptococcal disease. $N$ Engl f Med 1993;328:1843-

3 Fischer G, Horton RE, Edelman R. Summary of the National Institutes of Health Workshop on group B streptococcal infection. $\mathcal{F}$ Infect Dis 1983;148:163-6.

4 Feldman RG, Fleer A. The immune response to the group B streptococcus. Rev Med Microbiol 1992;3:52-8.

5 Yow MD, Mason EO, Leeds LJ, Thompson PK, Clark DJ, Gardner SE. Ampicillin prevents intrapartum transmission of group $\mathrm{B}$ streptococcus. $\mathcal{F} A M A \quad 1979 ; 241$ 1245-7.

6 Boyer KM, Gotoff SP. Prevention of early-onset neonata group B streptococcal disease with selective intrapartum chemoprophylaxis. $N$ Engl F Med 1986;314:1665-9.

7 Boyer KM, Gadzala CA, Kelly PD, Gotoff SP. Selective intrapartum chemoprophylaxis of neonatal group B streptococcal early-onset disease. III. Interruption of mother-to-infant transmission. F Infect Dis 1983;148: 810-5.

8 Morales WJ, Lim DV, Walsh AF. Prevention of neonatal group $B$ sepsis by the use of a rapid screening test and selective intrapartum chemoprophylaxis. Am $\mathcal{f}$ Obste Gynecol 1986;155:979-83.

9 Easmon CSF, Hastings MJG, Deeley J, Bloxham B, Rivers RPA, Marwood R. The effect of intrapartum chemoprophylaxis on the vertical transmission of group B streptococci. Br 7 Obstet Gynaecol 1983;90:633-5.

10 American Academy of Pediatrics, Committee on Infectious Diseases, Committee on the Fetus and Newborn Guidelines for prevention of group B streptococcal (GBS) infection by chemoprophylaxis. Pediatrics 1992;90:775-8.

11 Regan JA, Klebanoff MA, Nugent RP. The epidemiology of group B streptococcal colonization in pregnancy. Obstet Gynecol 1991;77:604-10.

12 Yancey MK, Armer T, Clark P, Duff P. Assessment of rapid identification tests for genital carriage of group B streptococci. Obstet Gynecol 1992;80:1038-47.

13 Islam AKMS. Rapid recognition of group-B streptococci. Lancet 1977; i:256-7.

14 Quentin R, Dubarry I, Gignier C, Saulnier M, Pierre F, Goudeau A. Evaluation of a rapid latex test for direct detection of Streptococcus agalactiae in various obstetrical and gynaecological disorders. Eur $\mathcal{f}$ Clin Microbiol Infect $D$ is 1993;12:51-4.

15 Green M, Dashefsky B, Wald ER, Laifer S, Harger J, Guthrie $R$. Comparison of two antigen assays for rapid intrapartum detection of vaginal group B streptococcal intrapartum detection of vaginal group B st

16 Stiller RJ, Blair E, Clark $P$, Tinghitella T. Rapid detection of vaginal colonization with group B streptococci by means of latex

17 Teti G, Burdash NM, Corey K, Fava C. Detection of group B streptococci by agglutination testing from selective broth cultures. Ann Clin Lab Sci 1985;15:292-8.

18 Jones DE, Friedl EM, Kanarek KS, Williams JK, Lim DV. Rapid identification of pregnant women heavily colonized with group B streptococci. $\mathcal{F}$ Clin Microbiol 1983;18:558-60.

19 Tuppurainen A, Hallman M. Prevention of neonatal group B streptococcal disease: intrapartum detection and chemoprophylaxis of heavily colonized parturients. chemoprophylaxis of heavily

20 Gentry YM, Hillier SL, Eschenbach DA. Evaluation of a rapid enzyme immunoassay test for detection of group $B$ Streptococcus. Obstet Gynecol 1991;78:397-401.

21 Armer T, Clark P, Duff P, Saravanos K. Rapid intrapartum detection of group B streptococcal colonization with an enzyme immunoassay. Am 7 Obstet Gynecol 1993;168:39-43.

22 Jones DE, Kanarek KS, Lim DV. Group B streptococcal colonization patterns in mothers and their infants. $f$ Clin Microbiol 1984;20:438-40.

23 Boyer KM, Gadzala CA, Kelly PD, Burd LI, Gotoff SP. Selective intrapartum chemoprophylaxis of neonatal group B streptococcal early-onset disease. II. Predictive value of prenatal cultures. F Infect Dis 1983;148: 802-9. 